\title{
Persistence of neurological damage induced by dietary vitamin B-12 deficiency in infancy
}

\author{
Ursula von Schenck, Christine Bender-Götze, Berthold Koletzko
}

\begin{abstract}
A case is reported of a 14 month old boy with severe dietary vitamin B-12 deficiency caused by his mother's vegan diet. Cinical, electroencephalography (EEG), and haematological findings are described. Cranial magnetic resonance imaging (MRI) showed severe frontal and frontoparietal cranial atrophy. Vitamin B-12 supplements led to a rapid improvement of haematological and neurological symptoms. Serum vitamin B-12 and urinary methylmalonate excretion were normal 10 days after treatment began. After six weeks, EEG was normal and cranial MRI after 10 weeks showed complete disappearance of all structural abnormalities. Cognitive and language development, however, remained seriously retarded at the age of 2 years. It is concluded that infantile vitamin B-12 deficiency induced by maternal vegan diets may cause lasting neurodisability even though vitamin $B-12$ supplementation leads to rapid resolution of cerebral atrophy and electroencephalographic abnormality.

(Arch Dis Child 1997;77:137-139)
\end{abstract}

Keywords: cobalamine deficiency; vegetarian diets; vegan diets; psychomotor development

Strict vegetarian diets with avoidance of milk and egg (vegan diets) do not provide appreciable amounts of vitamin B-12, because only foods of animal origin are significant dietary sources of the vitamin. Adults may tolerate vitamin B-12 deficient diets for many years without apparent symptoms due to their endogenous stores. In contrast, infants have very limited hepatic reserves of vitamin B-12 and so may develop symptoms of deficiency within months. ${ }^{1}$ Infantile vitamin B-12 deficiency with ineffective haematopoiesis and degeneration of nervous tissue has been reported in breast fed infants of mothers on strict vegetarian diets. ${ }^{2-7}$ Although vitamin B-12 supplements were reported to result in rapid improvement, ${ }^{89}$ concern has been raised regarding long term development. ${ }^{36710} \mathrm{We}$ report a case of a 14 month old boy in whom severe dietary vitamin B-12 deficiency caused persistent neurological damage.

\section{Case report}

The patient was referred to the Kinderpoliklinik, University of Munich, Germany at the age of 14 months because of anaemia and severe neurological abnormalities. His mother had been a vegetarian for 14 years and for the past six years she had followed a strict vegan diet with a high proportion of uncooked foods prepared according to the rules of Burger's raw food therapy. ${ }^{11}$ The patient was exclusively breast fed until the age of 9 months, when his mother started to offer him dried fruits and bananas. He took only small amounts of these complementary foods, however, for example, one date per day. His mother reported that he developed normally during his first 10 months; he could sit and walk with his hand held at 8 and 10 months respectively. From 12 months onwards, he gradually became less active and lost the ability to walk. By the age of 14 months, he was unable to sit, became increasingly apathetic, and eventually stopped feeding, which provoked referral.

On admission he was comatose, had a high pitched cry and severe generalised muscular hypotonia. He was malnourished with a length of $70 \mathrm{~cm}(4 \mathrm{~cm}<3 \mathrm{rd}$ centile $)$ and a weight of $7.3 \mathrm{~kg}(1 \mathrm{~kg}<3 \mathrm{rd}$ centile. A blood count showed macrocytic anaemia (haemoglobin 82 $\mathrm{g} / \mathrm{l}$, packed cell volume 0.245 , mean corpuscular volume $116.7 \mathrm{fl})$. Serum ferritin was 4.0 $\mu \mathrm{g} / \mathrm{l}$, and red blood cell folate $2018.1 \mathrm{nmol} / 1$. Serum vitamin B-12 was undetectable, and markedly increased urinary excretion of methylmalonate (32.4 mmol/g creatinine) confirmed vitamin B-12 deficiency. The concentration of vitamin B-12 in his mother's breast milk was $0.04 \mu \mathrm{g} / 100 \mathrm{~g}$. Samples from four healthy omnivorous mothers had a range of $0.11-12 \mu \mathrm{g} / 100 \mathrm{~g}$ (Dr W Schmolck, Milchwirtschaftliche Untersuchungs und Versuchsanstalt, Kempten, Germany).

Electroencephalography (EEG) on admission was severely abnormal with absent basal rhythmic activity. Cranial magnetic resonance imaging (MRI) showed severe frontal and frontoparietal cerebral atrophy (see fig 1A), and myelinisation was considered appropriate for age.

On admission, he was prescribed $250 \mu \mathrm{g}$ of vitamin B-12 intramuscularly, after which he received oral treatment with $25 \mu \mathrm{g}$ vitamin B-12/day and supplemental feeding with a soy formula, and rapidly improved. He regained consciousness within hours of receiving vitamin supplements. On the next day, he smiled and interacted with his mother for the first time in several weeks, his muscle tone improved, and he achieved head control. He regained the ability to walk. By the third day he was able to eat and drink and was discharged. His parents insisted that he continued a vegan diet but agreed to give him soy formula and oral vitamin B-12 supplements. 

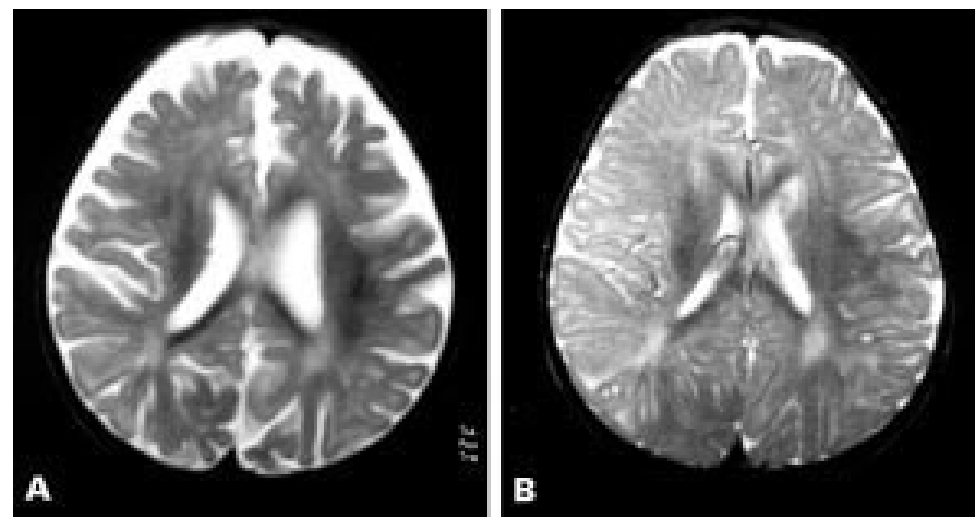

Figure 1 (A) Cranial MRI recording at the time of hospital admission shows severe frontal and frontoparietal cerebral atrophy, while myelinisation is considered normal for age, and (B) 10 weeks after the start of vitamin B-12 treatment the MRI shows complete disappearance of all structural alterations.

Serum vitamin B-12 and urinary methylmalonate excretion were normal 10 days after onset of treatment and at later follow up visits. Six weeks after treatment was begun, his EEG was normal. A cranial MRI 10 weeks after treatment started showed all structural abnormalities had disappeared (see fig 1B). Clinical follow up, however, showed persistent neurological damage. At age 17 months, he was inactive and showed no interest in playing. $\mathrm{He}$ could sit but remained hypotonic. At 2 years of age, he could walk, climb steps, and build a tower of four cubes, but still showed marked psychomotor retardation, was very agitated, and had poor concentration. He imitated sounds but had no recognisable words.

His two older sisters had no clinical signs of vitamin B-12 deficiency. Vitamin B-12 levels were normal in the 7 year old girl, who ate dairy products regularly and meat occasionally when not at home. The 3 year old girl had subclinical vitamin B-12 deficiency (103.3 pmol/1 serum). Serum ferritin and iron were reduced in both girls. Their mother had a reduced serum vitamin B-12 of $110.7 \mathrm{pmol} / 1$ and a ferritin of $21 \mu \mathrm{g} / 1$. Since her serum vitamin B-12 became normal after oral supplements we did not perform absorption studies.

\section{Discussion}

Infantile vitamin B-12 deficiency was first reported in six South Indian infants who presented at 7 to 12 months with megaloblastic anaemia, developmental regression, and skin hyperpigmentation. ${ }^{5}$ Five suffered from apathy and involuntary movements. All abnormalities disappeared within a few days of taking vitamin B-12 supplements; their long term development was not reported. Vitamin B-12 absorption was normal in all these infants, who had been breast fed by mothers with vitamin B-12 deficiency and low breast milk vitamin B-12 concentrations due to pernicious anaemia $(n=4)$ or vegan diets $(n=2)$. Several other reports on infantile vitamin B-12 deficiency have been published (see table 1). In eight infants, deficiency resulted from maternal pernicious anaemia, ${ }^{351012}$ in one it was due to maternal gastric bypass operation with ineffective secretion of intrinsic factor, ${ }^{13}$ while in 14 infants (including our patient) the cause was a maternal vegan diet (table 1$).^{2-46}$ Most presented with haematological and neurological symptoms at the age of 6 to 15 months. Michaud et al detected two clinically asymptomatic infants with very low plasma vitamin B-12 concentrations even at 2 and 3 months, respectively, when hyperaminoaciduria and increased methylmalonate excretion were detected on screening. ${ }^{12}$ Neither had haematological nor neurological changes and both developed normally with vitamin B-12 supplements.

The apparent morphological changes of neural tissues induced by vitamin B-12 deficiency resolve rapidly with treatment. Atrophy of the optic nerve resolved completely after six months of treatment. ${ }^{8}$ Stollhoff and Schulte reported a case of a patient with severe neurological changes, abnormal EEG, and marked frontal lobe atrophy on cranial computed tomography (CCT). ${ }^{6}$ Vitamin B-12 treatment resulted in rapid clinical response, a normal EEG after five weeks, and a normal CCT after 10 weeks.

There are limited data on long term development after severe neurological changes in infantile vitamin B-12 deficiency. Pearson and Turner followed up a child diagnosed at 32 months and found a Stanfort Binet IQ of 60 at the age of 6 years. ${ }^{10}$ Two of four children treated for infantile vitamin B-12 deficiency showed mild intellectual retardation on psychometric testing at the ages of 4 and 5

Table 1 Case reports of infantile vitamin B-12 deficiency in the literature

\begin{tabular}{|c|c|c|c|c|c|c|c|}
\hline Author & $\begin{array}{l}\text { No of } \\
\text { patients }\end{array}$ & $\begin{array}{l}\text { Aetiology of } \\
\text { vitamin B-12 } \\
\text { deficiency }\end{array}$ & $\begin{array}{l}\text { Age at } \\
\text { diagnosis } \\
\text { (months) }\end{array}$ & $\begin{array}{l}\text { Haemoglobin } \\
(<100 \mathrm{~g} / \mathrm{l})\end{array}$ & $\begin{array}{l}\text { Neurological } \\
\text { symptoms }\end{array}$ & Comatose & Neurodevelopment at follow up \\
\hline Jadhav et $a \bar{l}$ & 6 & $4 \mathrm{MPA}, 2$ vegan & $7-12$ & $6 / 6$ & $6 / 6$ & $5 / 6$ & No follow up \\
\hline Pearson and Turner ${ }^{10}$ & 1 & MPA & 32 & $1 / 1$ & $1 / 1$ & $1 / 1$ & Abnormal at 6 years \\
\hline Wighton et al & 1 & Vegan & 9 & $1 / 1$ & $1 / 1$ & $1 / 1$ & Abnormal at 12 years \\
\hline Higginbottom et al ${ }^{4}$ & 1 & Vegan & 6 & $1 / 1$ & $1 / 1$ & $1 / 1$ & No follow up \\
\hline Close $^{20}$ & 1 & Vegan & 12 & $1 / 1$ & $1 / 1$ & $1 / 1$ & No follow up \\
\hline Sklar ${ }^{9}$ & 1 & Vegan & 7 & $1 / 1$ & $1 / 1$ & - & Normal at 13 months \\
\hline Stollhoff and Schulte ${ }^{6}$ & 1 & Vegan & 14 & $1 / 1$ & $1 / 1$ & $1 / 1$ & Abnormal at 26 months \\
\hline McPhee et $a l^{14}$ & 1 & MPA & 5 & $1 / 1$ & $1 / 1$ & $0 / 1$ & Normal at 8 years \\
\hline Kühne $e t a t^{\beta}$ & 1 & Vegan & 9 & $1 / 1$ & $1 / 1$ & $1 / 1$ & Normal at 13 months \\
\hline Michaud et al ${ }^{12}$ & 2 & 1 MPA, 1 vegan & 2 & $0 / 2$ & $0 / 2$ & $0 / 2$ & Normal at 14 months and 2 years \\
\hline Graham $e t a l^{3}$ & 6 & $3 \mathrm{MPA}, 3$ vegan & $8-15$ & $4 / 6$ & $6 / 6$ & $6 / 6$ & $\begin{array}{l}2 \text { normal at } 9 \text { and } 10 \text { years, } \\
2 \text { abnormal at } 4 \text { and } 5 \text { years }\end{array}$ \\
\hline Almadan et $a l^{2}$ & 2 & Vegan & 5,24 & $2 / 2$ & $2 / 2$ & $2 / 2$ & No follow up \\
\hline Grange and Finlay ${ }^{13}$ & 1 & Gastric bypass & 10 & $1 / 1$ & $1 / 1$ & $1 / 1$ & Normal at 12.5 months \\
\hline Our patient & 1 & Vegan & 14 & $1 / 1$ & $1 / 1$ & $1 / 1$ & Abnormal at 26 months \\
\hline
\end{tabular}


years respectively; language delay was recorded in one. ${ }^{3}$

A diet devoid of vitamin B-12 usually does not induce clinical deficiency in adults for many years, because the average body stores of about $3 \mathrm{mg}$ balance the daily losses of about 3 $\mu \mathrm{g}$, and clinical symptoms of deficiency manifest themselves only when body stores are severely depleted. In contrast, newborn infants have body stores of about $25 \mu \mathrm{g}$ vitamin B-12. ${ }^{1}$ During the first month of life, about $0.1 \mu \mathrm{g}$ vitamin B-12/day are needed for tissue synthesis. ${ }^{14}$ Disregarding losses, we estimate that the body store of a normal newborn may last for eight months. Endogenous stores may be much lower if the baby's mother is vitamin B-12 deficient, ${ }^{15}$ which explains why Michaud et al found biochemical signs of infantile vitamin B-12 deficiency by 2 months of age. ${ }^{12}$ Vitamin B-12 and folic acid are essential cofactors for methionine synthesis and thus for formation of tetrahydrofolate required for methylation reactions in various metabolic pathways, including DNA synthesis. ${ }^{10}$ Typical neurological symptoms of infantile vitamin B-12 deficiency are cerebral and optic nerve atrophy, apathy and coma, hypotonia, and developmental delay. ${ }^{6}$ The molecular basis for these alterations is not well understood.

The primary function of cobalamin $(\mathrm{Cbl})$ is to provide coenzyme activity for the synthesis of methionine and succinyl-coenzyme A. For methionine synthesis, a methyl group is transferred from methyltetrahydrofolate (THF) to methyl-Cbl. The methyl transfer to homocysteine finally generates methionine. Thus two products essential for DNA synthesis are rendered: methionine and THF. THF becomes formyl-THF and provides C-1 units in purine synthesis. The absence of neurological symptoms in folate deficiency suggests that methionine synthesis may not be causally related to $\mathrm{Cbl}$ associated neuropathy. Attention has been focused therefore on the other $\mathrm{Cbl}$ dependent reaction: conversion of methylmalonyl-CoA to succinyl-CoA. An inappropriate methylmalonyl-CoA conversion results in an excess of the precursor propionyl-CoA. Propionyl-CoA accumulation leads to odd chain fatty acid synthesis, which leads to incorporation of large amounts of unusual C15 and C17 fatty acids in nerve sheets with altered neural functions. ${ }^{16}$

Cooper and Rosenblatt claim that deficiency of methionine synthesis causes the neuropathy induced by vitamin B-12 deficiency, ${ }^{17}$ and neurological damage has been described in disturbed methionine synthesis ${ }^{18}$ but not in isolated methylmanoyl-CoA mutase deficiency. ${ }^{17}$ Allen et al suggest that a deficiency in a third unknown $\mathrm{Cbl}$ dependent reaction is responsible for $\mathrm{Cbl}$ associated neuropathy, ${ }^{19}$ but the mechanism remains to be elucidated. Although vitamin B-12 treatment results in rapid resolution of haematological symptoms and morphological changes in the nervous system detectable by fundoscopy and imaging, there is no information regarding biochemical and func- tional alterations of neuronal cells in vivo and other physiological functions that may be important for long term outcome. It is conceivable that the long term prognosis of infantile vitamin B-12 deficiency depends on the severity and duration of the deficient state. Of the 25 cases reviewed (see table 1), six patients diagnosed at a mean age of 10 months were found to develop normally at follow up while six others diagnosed at a mean age of 13 months showed persistent developmental delay. Initial improvement does not necessarily result in a favourable long term outcome (personal observation). ${ }^{3610}$

Efforts should be directed therefore to preventing deficiency in pregnant and breast feeding women on vegan diets and their infants. Urinary screeening for methylmalonic aciduria may be useful in this selected population to detect affected individuals. ${ }^{12}$ The approach to individuals following unusual diets needs to be careful and considerate, aiming at stepwise introduction of small, acceptable additions such as milk products or eggs. If dietary changes are not acceptable to parents, vitamin B-12 supplements are essential.

1 Friedrich W. Handbuch der Vitamine. Munich: Urban and Schwarzenberg, 1987.

2 Almadan MS, al Awamy BH, al Mulhim IA. Nutritional vitamin B-12 deficiency in infancy. Indian $\mathcal{f}$ Pediatr 1993;60:683-5.

3 Graham SM, Arvela OM, Wise GA. Long-term neurological consequences of nutritional vitamin B-12 deficiency in infants. F Pediatr 1992;121:710-4.

4 Higginbottom MC, Sweetman L, Nyhan WL. Syndrome of methylmalonic aciduria, homocystinuria, megaloblastic anemia and neurological abnormalties in a vitamin B-12 deficient breast-fed infant of a strict vegetarian. $N$ Engl $\mathcal{F}$ Med 1978;299:317-23.

5 Jadhav M, Webb JKG, Vaishnava S, Baker SJ. Vitamin B-12 deficiency in Indian infants. Lancet 1962;iii:903-7.

6 Stollhoff K, Schulte FJ. Vitamin B-12 and brain development. Eur F Pediatr 1987;146:201-5.

7 Wighton M, Manson J, Speed I. Brain damage in infancy and dietary vitamin B-12 deficiency. Med f Aust 1979;ii:13 .

8 Kühne T, Bubl R, Baumgartner R. Maternal vegan diet causing a serious infantile neurological disorder due to vitamin B-12 deficiency. Eur f Pediatr 1991;150:205-8.

9 Sklar R. Nutritional vitamin B-12 deficiency in a breast fed infant of a vegan-diet mother. Clin Pediatr (Phila) 1986;25: 219-21.

10 Pearson AGM, Turner AJ. Folate dependent 1-carbon transfer to biogenic amines mediated by methylenetetrahydrofolate reductase. Nature 1975;258:173-4.

11 Burger GC. Die Rohkosttherapie. Munich: Heyne Verlag, 1976.

12 Michaud JL, Lemieux B, Ogier H, Lambert MA. Nutritional vitamin B-12 deficiency: two cases detected by routine new-born urinary screening. Eur $\mathcal{F}$ Pediatr 1992;151: tine new- 20 .

13 Grange DK, Finlay JL. Nutritional vitamin B-12 deficiency in a breastfed infant following maternal gastric bypass. Pediatr Hematol Oncol 1994;11:311-8.

14 McPhee AJ, Davidson GP, Leahy M, Beare T. Vitamin $B_{12}$ deficiency in a breast fed infant. Arch Dis Child 1988;63: 921-3.

15 Willoughby MLN. Vitamin B-12 deficiency. In: Willoughby MLN, ed. Pediatric haematology. Edinburgh: Churchill Livingstone, 1977:35-42.

16 Frenkel E. Abnormal fatty acid metabolism in peripheral nerve of patients with pernicious anaemia. $\mathcal{F}$ Clin Invest 1973;52:1237-45.

17 Cooper BA, Rosenblatt DS. Inherited defects of vitamin B12 metabolism. Annu Rev Nutr 1987;7:291-320.

18 Carmel R, Wathuis D, Goodmann SI, Rosenblatt DS. Heriditary defect of cobolamin metabolism (CblGmutation) presenting as a neurological disorder in childmutation) presenting as a neurological
hood. N Engl F Med 1988;318:1738-41.

19 Allen RH, Stabler SP, Savage DG, Lindenbaum J. Metabolic abnormalities in cobalamin (vitamin B12) and folate deficiency. FASEB F 1993;7:1344-53.

20 Close GC. Rastafarianism and the vegans' syndrome. BMF 1983;286:473. 